\title{
Tangence
}

\section{De quelques modulations et usages du fait divers}

\section{Gaston Lillo}

Numéro 37, septembre 1992

Autopsie du fait divers

URI : https://id.erudit.org/iderudit/025722ar

DOI : https://doi.org/10.7202/025722ar

Aller au sommaire du numéro

Éditeur(s)

Tangence

ISSN

1189-4563 (imprimé)

1710-0305 (numérique)

Découvrir la revue

Citer cet article

Lillo, G. (1992). De quelques modulations et usages du fait divers. Tangence, (37), 16-28. https://doi.org/10.7202/025722ar d'utilisation que vous pouvez consulter en ligne.

https://apropos.erudit.org/fr/usagers/politique-dutilisation/ 


\title{
De quelques modulations et usages du fait divers
}

\author{
Gaston Lillo*
}

I

Bien que la place réservée au fait divers dans le marché des biens symboliques ne soit pas particulièrement respectable et que cette pratique discursive ait plutôt une résonance péjorative, il n'en reste pas moins qu'elle jouit d'une énorme popularité auprès du vaste public de lecteurs d'informations de nos sociétés de consommation. Le lecteur des classes dominées, qui affiche ouvertement son attirance pour le fait divers, tout comme celui des classes dominantes, qui, lui, l'occulte comme une honte, consomment indistinctement ce type de récits, à tel point qu'il est difficile d'imaginer aujourd'hui un quotidien de la presse écrite ou même un journal télévisé qui ne consacre pas un espace, aussi petit soit-il, à la chronique du fait divers ${ }^{1}$.

Dès ses origines, dans le domaine de l'oralitê, le fait divers apparaît lié à la diffusion des nouvelles. Plus tard, avec la parution de la littérature de colportage, il s'incorpore et participe directement aux processus de formation de la presse moderne. Au cours de la deuxième moitié du $\mathrm{XIX}^{e}$ siècle et de la première du $\mathrm{XX}^{\mathrm{c}}$, ce type d'informations atteint une indiscutable hégémonie dans le monde des publications dites "populaires" (les " canards " français, les journaux à caractère sensationaliste, etc.) pour occuper enfin une place de choix dans les espaces informatifs des moyens audiovisuels: radio, télévision et cinéma.

Au delà de l'espace journalistique, les origines du fait divers se confondent avec celles de la littérature et de la culture dite populaire avec laquelle il entretient des rapports d'influence. C'est ainsi qu'on le retrouve dans la fête foraine et qu'il est repris,

* Université de Montréal, Groupe Marges.

1 Dans ce même ordre d'idées, il est intéressant de noter les observations d'un propriétaire d'un journal spécialisé dans le fait divers se plaignant de constater "que la presse "régulière" a envahi leur champ d'activités", André Noël, "Propagande haineuse. La censure ferait plus de mal que de bien *, La presse, 15 aôut 1992, p. B4. 
encore aujourd'hui, sous une forme fictionnalisée, par la chanson ${ }^{2}$ et le cinéma ${ }^{3}$. La littérature dite *culte * n'échappe pas non plus à son attraction. Nombreux sont les auteurs qui puisent leurs anecdotes dans ce que d'aucuns ont convenu d'appeler "les annales des passions excessives " 4 .

Pour la psychanalyse, le fait divers se présente comme un sujet d'étude particulièrement intéressant dans la mesure où il est généralement perçu comme le récit d'une anomalie, d'une transgression qui se produit toujours chez l'Autre, pouvant fonctionner ainsi à la manière d'une projection qui permet au lecteur/spectateur (sujet d'interpellation) de vivre par procuration ses fantasmes interdits et réprimés dans la société. Pour le sociologue, c'est son caractère marginal par rapport au discours journalistique, ses relations avec l'hégémonie discursive et la fascination qu'il exerce sur les masses dont il contribue à régler les modes de comportement et les valeurs, qui font du fait divers un objet d'étude.

Son contenu (crimes, monstruosités, catastrophes, faits insolites) et sa rhétorique (pacte de vraisemblance, utilisation stratégique du stéréotype) permettent de révéler non seulement les fantasmes, refoulements, peurs, désirs, etc., d'une collectivité, mais aussi les conflits qui la traversent à un moment donné de son histoire. La récurrence de sa thématique, de ses dramatis personae ainsi que d'une même structure logique (dominée par deux types de relations: la causalité troublée et la coïncidence ${ }^{5}$ ), ne constitue pas, à

2 Un exemple digne d'étude serait la saga de Pedro Navajas, délinquant fictif des rues de New York, mise en chanson par le chanteur portoricain Rubén Blades.

3 Dès ses débuts, le cinéma s'est intéressé au fait divers. Un des films les plus célèbres de l'époque du muet est Histoire d'un crime (1901) de Ferdinand Zecca qui est la reconstitution d'un crime assez curieux: une femme qui fait assassiner son amant par son mari. Méliès, pour sa part, s'inspire des catastrophes naturelles et des accidents pour réaliser Collision et naufrage en mer et Éruption volcanique à la Martinique. Les films d'Alice Guy, Les exécutions capitales (1904), Les Apaches de Paris (1905) et La danse béroïque (1914), sont aussi des adaptations de faits divers de son époque. Plus proches de nous, plusieurs films s'intéressent à des faits divers précis, par exemple: Moi Pierre Rivière de René Allio, El chacal de Nabueltoro de Miguel Littin, Cordélia (1979) de Jean Beaudin.

4 Il s'agit du sous-titre du numéro spécial consacré au faits divers par la revue Autrement, $\mathrm{n}^{\circ} 98$ (avril 1988).

5 Roland Barthes, "Structure du fait divers ", Essais critiques, Paris, Seuil, 1966. 
notre avis, une raison (valable) pour isoler le fait divers de son contexte historique de production en privilégiant une interprétation qui le renvoie exclusivement *aux constantes profondes de la culture et de l'inconscient populaire [...] aux archétypes qui [aux marges de l'histoire] hantent l'esprit humain ${ }^{6}$. Le fait divers qui rapporte l'assassinat perpétré par Marc Lépine, par exemple, dont le délire s'était cristallisé sur 14 femmes à l'École polytechnique de l'Université de Montréal, et surtout le caractère sélectif du geste (l'assassin ne tirait que sur des femmes), en disent long sur les tensions d'une société où les femmes en viennent à occuper des positions dans des domaines jusque-là réservés exclusivement aux hommes.

Le rapport entre ce type de récits et les contextes sociaux d'où ils émanent peut être également mis en lumière par un autre exemple (tiré de La presse du 8 août dernier) qui appartient à un tout autre registre - rappelons que la pratique discursive du fait divers couvre un vaste éventail allant du comique le plus désopilant au tragique ${ }^{7}$. Titrée *Oui à la fellation, non à la cigarette *, la chronique raconte l'arrestation, en Angleterre, d'un couple accusé d'avoir fumé une cigarette dans un wagon de train pour non fumeurs. Ce qui rend ce fait divers particulièrement cocasse, c'est que les amoureux avaient allumé la cigarette "pour se remettre de leurs ébats" après avoir consommé un acte sexuel oral en plein wagon, chose qui vraisemblablement n'avait pas dérangé les voyageurs qui n'ont cru bon d'alerter la police que lorsque l'incident de la cigarette s'est produit. Évidemment les élements retenus par le récit ne sont pas indépendants des campagnes anti-tabac, et de l'attitude du public face à des actes qui, dans un autre contexte, auraient été considérés comme des outrages à la pudeur. En effet, tandis qu'à l'époque victorienne la fellation était considérée comme une perversion 8 et la cigarette comme un signe de distinction, aujourd'hui, semble nous

6 Alain Monestier, Le fait divers, Musée national des arts et traditions populaires, Paris, Éditions de la Réunion des Musées nationaux, 1982.

7 En passant par le tragicomique. Par exemple: *Suicide manqué de 27 Chinois. * Manille 14 janvier 1982 (A.F.P.): Vingt-sept Chinois qui tentaient à Manille de s'immoler par le feu plutôt que de regagner la Chine ont raté leur suicide parce qu'ils avaient pris de la sauce de poisson pour de l'essence.

8 Dans un chapitre de son Trois essais sur la théorie de la sexualité consacré aux *aberrations sexuelles*, Freud affirme: "L'usage de la bouche comme organe sexuel est considéré comme perversion lorsque les lèvres (ou la langue) entrent en contact avec les parties génitales du partenaire; mais non lorsque les muqueuses buccales de deux partenaires se touchent* (trad. Reverchon-Jouve), Paris, Gallimard, 1962, p. 36. 
dire sur le mode ironique ce fait divers, un renversement de valeurs s'est produit: banalisation de la sexualité et "tabouisation" de la cigarette. Le récit de cet incident s'appuie donc sur des présupposés d'information contextuels et active une compétence communicative sans laquelle le lecteur aurait peine à décoder sa signification. Ce n'est pas que ce fait divers soit réactionnaire et propose un retour aux valeurs victoriennes, pas plus qu'il ne prône l'exercice public de la sexualité. On serait tenté de croire que ce qui est visé ici par l'ironie, c'est l'intolérance aux fumeurs. Mais l'énonciation est plus ambivalente et résiste à toute lecture qui lui attribuerait une prise de position univoque. Dès son titre, qui se présente d'ailleurs sous la forme d'un slogan évoquant la polémique qu'entraine de nos jours l'usage de la cigarette dans des lieux publics, l'énonciateur joue sur la bivocalité de l'énoncé. L'ironie du titre réside justement dans cette bivocalité. D'une part, le lecteur entend le contenu du slogan qui fonctionne comme une " citation " dont le contenu serait absurde et incohérent (même si curieusement les deux actes évoqués renvoient implicitement au même référent "bouche " et qu'une analogie entre la cigarette et le sexe masculin soit suggérée); d'autre part et en même temps, le lecteur entend le rire moqueur et distancié de l'énonciateur auquel il est appelé à s'identifier. Par le biais de plusieurs marques textuelles (il s'agit d'un "jeune couple *, de "deux tourtereaux ", qui s'offrent une "petite gâterie »), ce fait divers insiste avec humour sur le caractère paradoxal de la situation décrite et vise à railler le comportement d'une société en confirmant le stéréotype culturel du "flegme britannique". En effet, le public de ce wagon condamne et dénonce à la police la transgression de la loi qui interdit de fumer tandis qu'il reste imperturbable devant l'accomplissement d'une fellation sous ses yeux.

\section{II}

D'une façon générale, le type d'information contenue dans un fait divers ne prête guère à débat public et ne devient qu'exceptionnellement matière privilégiée d'un débat de société qui impliquerait des politiciens et d'autres instances de pouvoir. Parmi la masse des discours produits par une société, le fait divers semble prendre l'allure d'une rumeur. Une rumeur le plus souvent confinée à de courts entrefilets, qui ne rapporte pas les affaires "sérieuses " faisant la une de l'information, mais qui parle à sa manière des tensions sociales, politiques, culturelles de la collectivité et qui, comme un cri, jaillit quand la transgression à 
laquelle elle fait référence ébranle les fondements les plus chers d'une société. Tel fut le cas du fait divers (que nous avons évoqué plus haut) relatant la tuerie de Marc Lépine, qui a mobilisé tous les médias (écrits et électroniques). En s'attaquant à des membres de l'élite du corps social (du point de vue symbolique, ses victimes représentaient des aspirations et des valeurs fortes prisées socialement), son geste a déclenché plusieurs débats au sein des institutions (sur la marginalisation sociale, la santé mentale, le contrôle des armes, l'efficacité de la police, les revendications des femmes, etc). On se souviendra également d'une série de faits divers rapportés par les médias dans l'intervalle de quelques semaines relatant les abus sexuels perpétrés au Canada par des religieux sur de jeunes adolescents. Dans ce cas-ci, le passage d'une simple rumeur à un débat de société s'explique par la révélation en chaîne de faits semblables qui remplirent l'espace réservé aux faits divers et surtout parce que les attributs moraux que la société - majoritairement chrétienne - reconnaît aux membres des institutions religieuses étaient remis en question.

Ces remarques sur les conditions qui font d'un fait divers un événement notoire nous amènent à une réflexion sur la place qu'occupent les acteurs de ces faits divers dans la symbolique sociale. En effet, qu'en aurait-il été de la presse si le crime de Marc Lépine avait été commis sur la personne de jeunes ouvrières ou de prostituées? et si le cas de Gilles Perron, accusé d'avoir tué sa femme, n'avait pas impliqué des personnalités du monde de l'entreprise et des médias? Et si les agressions sexuelles n'avaient pas été commises par des religieux ou par des politiciens? Il en aurait sans doute été tout autrement. C'est donc aussi en fonction du prestige ou du rôle social des acteurs que le fait divers acquiert notoriété et peut quitter son habituelle position marginale et faire les grands titres. Des exemples comme ceux du président Bush accusé d'avoir eu des relations particulières avec une secrétaire; du ministre Charron, appréhendé pour vol à l'étalage; du député Untel ou du télévangéliste P. Lacroix, accusés d'abus sexuels sur des adolescents, déçoivent les attentes de comportement irréprochable que la société se croit en droit d'exiger d'eux.

Un autre élément déterminant en ce qui a trait à l'importance (la place?) que les médias accordent aux faits divers et à l'impact que ces derniers peuvent avoir au niveau de la réception, c'est la proximité physique et psychologique du fait divers avec le lecteur ou spectateur virtuel. Dans le cas particulier qui est à l'origine de 
ce numéro de revue, celui de l'adolescent qui tue à coups de hache son père, sa mère, un de ses frères et une de ses sœurs (étrange ressemblance avec le cas de Pierre Rivière commenté par Foucault), l'éloignement spatial du crime est certainement une des raisons qui a le plus contribué à le reléguer à une position secondaire dans le Journal de Montréal d'où il est tiré, même si son titre - Une famille massacrée à la hache en gros caractères souligne avec sensationnalisme la sauvagerie, l'horreur de l'acte. Cette distance joue un rôle sécurisant. C'est comme si le lecteur se disait: - ce n'est ni à nous, ni à un de nos proches que cela est arrivé ", .l'enfer c'est les autres. Mais, en même temps, la proximité psychologique que le fait divers établit avec son lecteur lui rend toute sa dimension paradoxale, car cette fois il est appelé à jouer un rôle menaçant: tous les éléments familiers qui rapprochent le lecteur de la situation présentée en font un éventuel protagoniste.

Dans l'exemple en cause, la présentation d'une photo des quatre membres de la famille, une famille parmi tarit d'autres, visiblement heureuse (les sourires renvoyant au sème /bonheur/), est l'élément essentiel du processus d'identification que ce fait divers vise à déclencher chez son lecteur ${ }^{9}$. Implicitement, la photo choisie et l'énoncé de la légende qui l'accompagne: *Quatre des membres de cette famille ont été exécutés présumément par celui qu'on aperçoit en haut, à droite ", tendent à établir que *cette famille * pourrait très bien être la vôtre.

Les éléments du quotidien qui complètent la description (cassette, écolier, etc.) ainsi que la motivation présumée du meurtre (l'adolescent ne voulait surtout pas que son père écoute une cassette qu'il venait d'acheter - ce qui correspond au principe de la causalité aberrante propre au fait divers: petite cause, grands effets ${ }^{10}$ ) participent aussi de cette dimension menaçante. L'allusion aux * vêtements de nuit * que portaient les victimes (même si *la police pense qu'ils ont été tués jeudi matin ") sert aussi à éveiller les sentiments de peur du lecteur. Dans l'imaginaire social, la nuit est associée à la mort et entourée de mystères et de superstitions: pendant le sommeil, on est à la merci de toutes les agressions possibles.

Par ailleurs, ce fait divers renvoie à la situation de crise d'adolescence qui fait partie du quotidien de bien de familles. Si, d'un

9 - Voir, c'est deviner dans un pli de visage tout un monde semblable au nôtre *, Maurice Merleau-Ponty, Signes, Gallimard, 1960, p. 388.

Roland Barthes, loc. cit. 
22

point de vue psychanalytique, pour devenir adulte, l'adolescent provoque la mort symbolique du père, dans ce cas-ci c'est cette mort symbolique qui devient mort réelle.

\section{III}

Une idée généralement acceptée est que le fait divers appartiendrait à la culture populaire, serait l'expression de l'imaginaire des gens du peuple, principaux consommateurs de ce type d'informations. Se référer au fait divers du point de vue de ses rapports avec la culture populaire demanderait un développement de la notion de culture populaire qui dépasserait le cadre de cet article. Il nous semble cependant difficile de ne pas indiquer, ne serait-ce que brièvement, notre position par rapport à cette notion complexe à laquelle nous adhérons. Nous tenons pour acquis que la notion de culture populaire comporte une dimension contestataire de l'ordre symbolique dominant et que c'est à l'intérieur des rapports discursifs conflictuels, tensions, oppositions qui caractérisent toute société, qu'il conviendrait de situer les enjeux théoriques qu'elle pose. Ainsi, la culture populaire serait à distinguer de la culture de masse dont la rentabilité commerciale et la fidélité au système dominant de représentations constituent les principales caractéristiques. Bien que cette position de contestation que nous attribuons à la culture populaire ne soit pas tout à fait indépendante des conflits entre groupes sociaux (dominants/dominés) elle dépasse la problématique des sujets qui y interviennent. Les consommateurs tout autant que les producteurs de la chronique des faits divers se trouvent à l'intérieur d'une symbolique sociale dont les déterminations vont au delà des intérêts conscients des uns et des autres.

$\mathrm{Si}$, à ses origines, le fait divers était une véritable création collective - le mode de diffusion orale encourageait les interventions du public qui ne se contentait pas d'un rôle passif et qui à son tour devenait sujet de l'énonciation de ces "textes " souvent réélaborés - , aujourd'hui, dans les sociétés dirigées, dans les sociétés de masses, "un système de plus en plus totalitaire ne laisse plus aux consommateurs une place pour marquer ce qu'ils font, ce qu'ils fabriquent avec les produits qui leur sont offerts" comme l'a souligné Michel de Certeau ${ }^{11}$. Le fait divers devient ainsi, du moins modulé sous sa forme la plus courante, c'est-à-

11 Michel De Certeau, L'invention du quotidien, Paris, UGE, 1976, [n.p.l. 
dire celle des journaux, des revues sensationnalistes et dernièrement des films et vidéos consacrés au crime, un produit de la culture de masse qui nous apparaît comme étant complémentaire à la culture hégémonique, fonctionnant comme variante misérable, dégradée, vulgarisée de cette culture ${ }^{12}$. C'est le cas par exemple des journaux comme Allo police, Photo police, Crimes et passions (Montréal) et des films et/ou vidéos L'Amérique interdite (vol. I et II), Faces of Death (vol. I, II, III et IV), L'Asie interdite, L'Amérique à nu, etc.

Bien qu'ils se présentent comme des produits "transgressifs", les seuls osant montrer ce que la société essaie d'occulter, activant des zones de l'imaginaire classées dans la catégorie du tabou, ces documents s'inscrivent en réalité dans une perspective plutôt conservatrice, moralisante et fortement attachée à l'ordre établi. Ce sont ces médias qui réclament avec le plus d'animosité des châtiments exemplaires pour les coupables ou applaudissent avec ferveur leur condamnation selon les cas, alimentant ainsi les fantasmes sadiques de leur public dont fait partie, nous ne l'ignorons pas, une grande majorité des policiers. L'emploi de qualificatifs tel que "chacal ", "bêten, "monstre " ou tout terme soulignant la sauvagerie de l'acte criminel comme "éventreur", "étrangleur", etc., renforce une condamnation sans appel et exclut toute possibilité de pardon ${ }^{13}$. Ces médias, par ailleurs, véhiculent souvent et vivifient les stéréotypes culturels, voire même raciaux ${ }^{14}$, que la presse dite sérieuse ne se permettrait pas d'affirmer par respect du décorum, même si, secrètement, elle les partage.

La dimension "contre-culturelle" de contestation de l'ordre symbolique dominant que nous attribuons à la notion de culture populaire est totalement exclue de la modulation dans laquelle le fait divers relatif au crime est énoncé par ces médias de la culture de masse.

12 À ce propos, voir Umberto Eco, Apocalípticos e integrados (trad. A. Boglar), Barcelona, Lumen, $6^{*}$ éd., 1981.

13 * En réclamant la rigueur de l'échafaud, le public cherche peut-être à éliminer symboliquement le désir meurtrier qu'il éprouve inconsciemment lui-même [...]. Manière d'abolir symboliquement le désir de meurtre qu'il pressent en soi, l'exécution capitale est peut-être aussi, pour le spectateur, ou pour le lecteur qui, à travers la chronique, en suit le déroulement, l'occasion de commettre fictivement ce même meurtre* Le fait divers, op. cit., p. 43.

14 Au cours du mois d'août 1992, le journal Pboto police est mis sur la sellette pour ses propos racistes envers la communauté noire du Québec. 
Dans certaines émissions de télévision (Urgences diffusée à Quatre saisons, Section Disparus à Télé-Métropole ou S.O.S en France, etc.), l'usage du fait divers répond principalement à un besoin chez le spectateur d'étancher sa soif de situations extrêmes impliquant souvent le danger de mort (accidents de la route, asphyxie, incendies, vols, agressions, etc.). La reconstitution de faits divers (avec des acteurs professionnels ou même les véritables protagonistes) a avant tout une visée didactique: donner aux téléspectateurs des rudiments de secourisme et d'autodéfense. Au delà de cette visée éducative, ces émissions exaltent de surcroît l'héroïsme, l'abnégation et une notion de civisme où la figure du représentant de la loi et des institutions est proposée au téléspectateur comme une image d'identification structurant ses contenus selon des oppositions radicales entre le bien et le mal. Sont utilisés des procédés tels que le suspens, l'attente anxieuse du happy end propre au mélodrame, etc. Le bappy end, produit grâce à l'intervention d'un héros dont la mission est de rétablir l'ordre transgressé, annonce l'épilogue du récit où l'acte d'hérö̈sme sera reconnu et récompensé publiquement par la remise d'un objet symbolique (médaille, diplôme, etc.).

Quant au cinéma de fiction, ce sont surtout le genre policier et le film d'horreur qui, visant surtout l'exacerbation des affects, puisent dans les chroniques du fait divers. Les renvois à l'extraordinaire, à l'insolite, font que ces deux *genres " de cinéma s'intéressent particulièrement à ce type de récits, contrairement à la comédie bourgeoise dont le pacte de vraisemblance empêche les altérations trop brusques de la logique régissant l'univers du quotidien.

On peut trouver cependant des films très sobres tirés directement d'un fait divers comme Moi Pierre Rivière de René Allio (récit d'un criminel qui avait fait l'objet d'une étude de Michel Foucault) ou El Chacal de Nabueltoro de Miguel Littin, un classique du cinéma latino-américain; Cordélia, L'affaire Coffin (JeanClaude Labrecque, 1973), pour ce qui est du cinéma québécois, mériteraient également des études sérieuses.

Depuis un certain temps déjà, un nouveau genre de films vient élargir le domaine déjà fort encombré des médias spécialisés dans la chronique des faits divers; ces films tirent profit à la fois du succès du fait divers dans les médias imprimés et de la popularité de la vidéo, forme sous laquelle ils sont principalement diffusés. À la différence de la modélisation du fait divers du journal 
télévisé, très proche de celle de la presse écrite, ce nouveau genre de films travaille une notion large du fait divers exploitant la tradition foraine de l'exhibition de bizarreries. Il devient ainsi une sorte de version cinématique du musée de l'horreur.

Même s'ils se donnent à lire/voir comme des "documentaires " (L'Amérique interdite), des "réflexions * sur le monde (Mondo Cane), ou encore comme des "études scientifiques" (Faces of Death), ces films doivent surtout leur succès commercial à l'attrait qu'exerce tout ce qui sort de l'ordinaire et qui concerne en particulier les transgressions des tabous sur la mort et la sexualité. On assistera par exemple à des scènes de combats féminins de boxe où les adversaires exhibent leurs seins, ou des combats de lutte où des femmes à moitié nues se roulent dans la boue. Quant aux scènes mettant l'accent sur la mort, on assistera par exemple à la mise à mort de condamnés à la chaise électrique ou à la chambre à gaz ${ }^{15}$; à la trépanation d'un singe encore vivant pour en savourer la cervelle, à une scène de dégustation de vers de terre et à toute une série de catastrophes et d'accidents violents.

Le discours en voix off qui accompagne les images et qui se veut selon les cas écologiste, pacifiste ou condamnatoire de la peine de mort, est non seulement scientifiquement mal documenté mais il reste bien en deçà de la jouissance scopique que procure le phénomène de l'horreur dans ce type de films. Il nous semble que ce discours sert plutôt de prétexte qui viendrait cautionner la monstration d'images morbides.

Ce n'est pas la dimension pseudo-scientifique ou écologiste du discours en voix off contenu dans ces films qui est la plus mise en valeur dans les paratextes publicitaires et dans les titres. $\mathrm{Au}$ contraire, ce qui ressort le plus fréquemment dans ces éléments paratextuels (les titres, les affiches de publicité ou les boîtes des vidéo-cassettes) appartient au champ sémantique de l'insolite et de l'interdit qui, comme nous l'avons déjà dit, piquent la curiosité du spectateur/lecteur, en l'invitant à vivre une expérience "unique" réservée exclusivement aux "initiés * (citation de la publicité).

15 Le genre renoue avec une pratique des temps où le cinéma était encore considéré comme spectacle forain, jusqu'à ce que le ministère de l'Intérieur interdise, en 1910, la projection d'exécutions capitales qui connaissait un trop grand succès. 
L'adjectif * interdit " des titres renvoie à une idée de subversion et propose les premiers termes du pacte de vraisemblance implicite entre le film et son spectateur qui est ainsi invité à violer une norme, à voir ce qui est généralement interdit au regard. Un autre élément de ce pacte implicite concerne l'adhésion à la réalité que réclament ces faits divers filmés. Une série de marques indiquent au spectateur que ce qu'il est en train de voir a "réellement - eu lieu quelque part à un moment donné, ce qui contribue à augmenter l'impact que celui-ci peut éprouver. Cependant, nous avons pu observer que plusieurs des cas présentés ne sont que de mauvaises reconstitutions qui sonnent faux (puisque le truquage est vite repéré) et trahissent sans la moindre dissimulation le pacte de vraisemblance.

$\mathrm{Si}$, dans les cas des prises de vue in vivo où les incidents sont filmés au moment même où il se produisent, les images confirment le pacte de vraisemblance "réaliste. (comme, par exemple, dans les cas des exécutions capitales, des catastrophes naturelles et des accidents), dans le cas des reconstitutions (présentés pourtant aussi comme du * cinéma direct "), le réalisme propre de l'image, paradoxalement, va à l'encontre de l'effet de réalité.

Ni l'aspect informatif ni la vérifiabilité de ce qui est présenté ne sont importants dans ces films: les données sont vagues et les généralisations stéreotypées, courantes.

\section{IV}

Étant donné que le processus de mise en discours et de reproduction (mise en boîte) est, dans le cas de l'industrie du cinéma et de la vidéo, plus lent et plus coûteux que dans la presse écrite et électronique, le fait divers dans le film n'est pas en mesure de répondre au caractère d'actualité, de "nouvelle " qu'il présente dans les autres médias. Dans la presse écrite, c'est une dépêche généralement envoyée par télex, la nuit précédente, par une agence d'informations qui assure son actualité. Dans le journal télévisé, le fait divers se réfère toujours à un passé immédiat ou, dans les cas de transmissions en direct, au présent; dans les deux cas, il est toujours daté.

Dans le genre le films que nous commentons, les récits renvoient soit à des événements qui ont eu lieu ponctuellement dans un passé relativement lointain, comme par exemple la mort d'un parachutiste, une catastrophe aérienne, etc., soit à des événements 
qui sont isolés de leurs occurrences réitérées (des pratiques rituelles de certaines sectes, les partys dans certains bars de New York, etc). Dans les deux cas, les faits divers sont traités à l'intérieur d'une thématique majeure (la mort, la violence, etc.) et servent à illustrer de manière stéréotypée le comportement de certaines cultures ou collectivités: par exemple, dans L'Amérique interdite, la liberté sexuelle des Américains, leur goût des armes, etc. Dans un autre exemple tiré de la série de films vidéo Faces of Death, on peut constater que la plupart des récits où il est question des cultures non occidentales présentent des traits discriminatoires envers elles (c'est le cas des Shuars, appelés Jivaros dans le film, qu'on qualifie à plusieurs reprises de "redoutables sauvages ").

$\mathrm{Si}$, dans le fait divers écrit, l'événement est * rapporté *, dans le fait divers filmique, le spectateur en est le témoin oculaire. La médiation de l'appareil énonciatif, surtout dans le registre "réaliste ", se fait presque invisible et l'expérience spectatorielle accomplit ce que Baudrillard appelle le "vertige de la présence totale à l'événement •; celui-ci rappelle aussitôt que, paradoxalement, "la vérité de la chose vue, télévisée, magnétisée sur bande, c'est précisément que je n'y étais pas.. Dans l'expérience spectatorielle du fait divers, c'est ce vertige qui l'emporte sur la présence elle-même; "ce que nous donnent les communications de masse ce n'est pas la réalité, c'est le vertige de la réalité . Et il ajoute:

La pratique des signes est toujours ambivalente, elle a toujours pour fonction de conjurer au double sens du terme: de faire surgir pour capter par des signes (les forces, le reel, le bonheur, etc.) et d'évoquer quelque chose pour le nier et le refouler. [...] D'une certaine façon, la consommation généralisée d'images, de faits, d'informations, vise elle aussi à conjurer le réel dans les signes du réel.

Dans la réception du fait divers (dont l'universalité, note Baudrillard, caractérise la société de consommation), *c'est le plus vrai que le vrai qui compte, autrement dit le fait d'y être sans y être, autrement dit encore le phantasme * ${ }^{16}$.

\section{V}

Que faire en définitive du fait divers, si ce n'est, profitant de l'excès et de l'insolence qui le caractérisent, une réutilisation

16 Jean Baudrillard, La société de consommation, ses mythes, ses structures, Paris, Denoël, 1970, [n.p.]. 
28

contestataire qui se joue à la fois du tabou, de l'interdit et du bon goût? Une telle lecture est de ce point de vue considérée comme une façon de braconner, de ruser avec les représentations en place.

Contre la célébration de l'excès, la recherche et la reproduction de pures sensations (et on a voulu insister sur le fait que c'est cette dimension qui, actuellement, est la plus exploitée par le fait divers), il est possible d'imaginer un spectateur qui échapperait à cette emprise et qui, avec créativité, construirait des espaces de lecture différents de ceux qui lui sont proposés par la culture de masse. Mais le système ne lui laisse guère de place où marquer ce qu'il fait réellement avec ces produits. Cette tâche incombe à l'écrivain, au cinéaste, au producteur de biens symboliques à l'écoute des aspirations culturelles de ceux qui résistent à l'ordre du discours. 